Sparing zeigte Daten einer bereits veröffentlichten Studie [Neurology 2012; 78: 24-30] bei 20 Patienten mit linksseitigem Neglect. Zehn erhielten eine linksseitige hemmende Magnetfeldtherapie, die übrigen zehn eine Scheintherapie mit geringen, biologisch unwirksamen Feldstärken. Alle nahmen zudem am üblichen Aufmerksamkeitstraining teil. Behandelt wurde zweimal täglich werktags über zwei Wochen hinweg, den Erfolg maßen die Ärzte anhand der Skala BIT (Behavioral Inattention Test). Das Ergebnis: Zwei Wochen nach Therapiebeginn war der BITWert mit der Scheintherapie nur von 170 auf 175 Punkte gestiegen, mit der echten Stimulation dagegen von 170 auf etwa 190 Punkte. Die Magnetfeldtherapie war damit deutlich besser als die alleinige Standardtherapie mit Aufmerksamkeitstraining. Anhand von evozierten Potenzialen konnten die Neurologen weiter feststellen, dass die Übererregbarkeit in der linken Hemisphäre in der Stimu-lationsgruppe deutlich zurückgegangen war, nicht aber in der Kontrollgruppe. Ein Manko: Die Verbesserung reichte nicht aus, damit die Patienten im Alltag selbstständiger wurden. Allerdings war der Effekt bei den am stärksten betroffenen Patienten auch am intensivsten, sodass die Magnetstimulation gerade bei schwerem visuellen Neglect eine nützliche Therapie sein könnte.

\section{Mentale Schreibmaschine für Locked-in-Patienten}

Auch für Patienten mit Locked-in-Syndrom, die nach einem Schlaganfall bis auf das Augenlid komplett gelähmt sind, ist noch eine gewisse Rehabilitation möglich: Für sie sind sogenannte Gehirn-Computer-Schnittstellen dann oft die einzige Möglichkeit, mit der Außenwelt zu kommunizieren. Spezielle Algorithmen erkennen dabei gedachte Bewegungen im EEG. Damit lässt sich am Computer schreiben: In einer halben Stunde können die Patienten alleine mit der Kraft ihrer Gedanken immerhin etwa 200 Buchstaben „tippen“, sagte Professor Gabriel Curio von der Charité in Berlin.

Bei dem Verfahren wird die Aktivierung des Kortex bei geplanten Bewegungen aufgezeichnet. Eine Software lernt dabei, während der etwa 15 bis 30 Minuten dauernden Kalibrierungsphase, diejenigen motorischen Signale herauszufil- tern, die für die jeweilige Bewegung, etwa eine Armbewegung, typisch sind. Mit der Vorstellung, einen Arm zu bewegen, lässt sich somit eine Art Cursor auf einem Buchstabenfeld manövrieren; der Patient kann dann den gewünschten Buchstaben auswählen. Selbst bei lange zurückliegenden Lähmungen treten motorische Signale auf geplante Bewegungen hin noch auf. Allerdings verlernt das Gehirn irgendwann, Potenziale für gelähmte Muskeln auszubilden, sodass die Übungsphase dann etwas länger dauert und die Signale nicht mehr ganz so deutlich sind, sagte Curio.

\section{Mit Gehirn-Computer-Schnitt- stellen wieder laufen können?}

In Zukunft könnten mit Gehirn-Computer-Schnittstellen auch Prothesen oder Exoskelette bedient werden, mit denen Gelähmte eines Tages vielleicht wieder per mentaler Steuerung gehen können. Allerdings, so räumte der Neurologe ein, hat die Methode ihre Grenzen: Die von außen abgeleiteten EEG-Potenziale bilden eher eine Art Hintergrundrauschen, damit könne man nur sehr grob erkennen, was im Gehirn passiert. So werde es zum Beispiel nicht möglich sein, einen Buchstaben zu erkennen, den sich jemand intensiv vorstellt. Eine bessere Auflösung versprechen etwa epidurale Elektroden, an denen ebenfalls geforscht wird. Sie könnten zur Steuerung von Prothesen deutlich besser geeignet sein.

An den abgeleiteten EEG-Potenzialen hat auch die Industrie Interesse. Damit ließe sich etwa das Verhalten von Menschen überwachen, die in sicherheitskritischen Bereichen tätig sind. Ein solches Gerät könnte etwa Alarm schlagen, wenn jemand, der ein Fahrzeug bedient, kurz vor dem Einschlafen ist.

Thomas Müller

56. Jahrestagung der Deutschen Gesellschaft für Klinische Neurophysiologie und funktionelle Bildgebung (DGKN) in Köln am 15. März 2012

\title{
Frühe Neuromodulation
}

Bisher bestehen für Schlaganfallpatienten jenseits der Akuttherapie meist nur physiotherapeutische Trainingsmaßnahmen zur Verfügung, um Folgen wie eine Sprachstörung oder eine Halbseitenlähmung zu lindern. In der Klinik und Poliklinik für Neurologie der Uniklinik Köln versucht man nun auf einer speziell eingerichteten Frührehabilitationsstation Patienten mit Hirnschädigungen durch ein interdisziplinäres Team aus Ärzten, Physiotherapeuten, Ergotherapeuten, Logopäden und Psychologen bereits in der Akutphase der Erkrankung individuell zu behandeln. Darauf wies Professor Gereon Fink, Kongresspräsident der 56. Jahrestagung der DGKN, hin.

Schlaganfallpatienten mit Lähmungen oder kognitiven Ausfallserscheinungen erhalten bereits in den ersten Tagen nach dem Ereignis eine gezielte Hirnstimulation, um die natürlichen Reorganisationsprozesse des Gehirns zu unterstützen. Hierbei reizen die Therapeuten per transkranieller Magnetstimulation oder transkranieller Gleichstromstimulation gezielt Kortexregionen, deren Funktion durch den Schlaganfall gestört ist. Dieser neue Ansatz zur Rehabilitation basiert auf jahrelangen Vorarbeiten, in denen gezeigt wurde, dass es in der Akutphase eines Schlaganfalls nicht nur zu Beeinträchtigungen in der unmittelbar betroffenen Hirnregion kommt, sondern dass dabei ganze Netzwerke gestört werden. Entsprechend lassen sich per nicht invasiver transkranieller Stimulation einzelne Hirnregionen in ihrer Aktivität verstärken, oder aber die Einflüsse hemmende Regionen schwächen. mut

56. Jahrestagung der Deutschen Gesellschaft für Klinische Neurophysiologie und funktionelle Bildgebung (DGKN) in Köln vom 15.-17. März 2012 